\title{
Review on Triclabendazole Resistance in Fasciola
}

\begin{abstract}
Keywords: Anthelmintics; Fasciola; Resistance; Triclabendazole Abstract

The control of Fasciolosis can be achieved by application of anthelmintic drugs, elimination of the number of intermediate hosts and reduction of exposure to infection. Triclabendazole, which is a member of Benzimidazole, is most recommended and effective way of controlling fasciolosis in animals and humans that can kill both mature (adult) and immature liver flukes. This drug have able to penetrate the tegument of Fasciola (F) hepatica by diffusion, and the fluke is able to sulfoxidate the drug to the active sulfoxide metabolite which binds to $\beta$-tubulin and thus inhibit the formation of microtubules that are components of cytoskeleton of the parasite. However, in recent year, resistance of Triclabendazole is reported in animals and humans in different regions of the world. Resistance has likely appeared due to a generally poor understanding of liver fluke biology by farmers and con-founding factors, such as incorrect dosing, inappropriate product choice, and lack of testing for efficacy. These conditions may lead to reduced diffusion and metabolism of the drug. change efflux pump activity and changes in the target molecule that can reduce the effectiveness of Triclabendazole. Both in-vivo and in-vitro methods, like Faecal Egg Count Reduction Test (FECRT) and the Egg Hatch Assay (EHA), respectively, can help to investigate the resistance of Triclabendazole. Administration of dual active flukicide drugs, development of vaccines, implementation of Fasciola control methods other than Triclabendazole, and use of accurate dosage at appropriate time can help to reduce the incidence of Triclabendazole resistance.
\end{abstract}

\section{Introduction}

Anthelmintics are drugs that are used to treat infections with parasitic worms. This includes both flat worms, e.g., flukes and tapeworms and round worms, i.e., nematodes. They are of huge importance for human tropical medicine and for veterinary medicine. Broad spectrum anthelmintics are effective against parasitic flat worms and nematodes [1].

Triclabendazole (TCBZ), benzimidazole derivative, is one of the major anthelminthic drugs used to control fasciolosis in domestic animals. Triclabendazole was first introduced as a flukicide during the early 1980s. It has an efficacious (> 98\%) drug for both mature and immature flukes and has been used to treat and control fasciolosis [2]. Due to its efficacy for immature flukes TCBZ is the best drug of choice among other anthelminthic agents and considered as an Achilles heel in the overall control of liver fluke [3]. This over-reliance on TCBZ to treat sheep and, to a lesser extent, cattle, has resulted in selection for flukes resistant to TCBZ [4]. The status of Triclabendazole-Resistance (TCBZ-R) in F. hepatica has been reviewed elsewhere [5].

Benzimidazoles (BZs) are effective against a broad range of parasites and also have wide safety margins, working at dosages of $\mathrm{mg} / \mathrm{kg}$ bodyweight [6]. Their mode of action appears to be mediated through binding to $\beta$-tubulin within the parasite, thus inhibiting the formation of microtubules that are central to the form and function of the parasite's cells. This prevents various essential cellular processes such as the transport of secretory granules and enzymes in the cell cytoplasm, resulting in cell lysis, with knock-on detrimental effects on motility and feeding [7].

\section{Journal of}

Veterinary Science \& Medicine

\author{
Warkaw Merachew ${ }^{1}$ and Tewodros Alemneh ${ }^{2 *}$ \\ ${ }^{\prime}$ School of Veterinary Medicine, College of Agriculture and \\ Veterinary Medicine, Jimma University, Jimma, Ethiopia \\ ${ }^{2}$ Woreta City Office of Agriculture and Environmental Protection, \\ South Gondar Zone, Amhara Regional State, Ethiopia
}

*Address for correspondence:

Alemeneh T, Expert Veterinarian at Woreta City Office of Agriculture and Environmental Protection, South Gondar Zone, Amhara Regional State, Ethiopia, Tel: 2519204998 20; Email: tedyshow@gmail.com

Submission: 05-August, 2020

Accepted: 21-September, 2020

Published: 25-September, 2020

Copyright: $\odot 2020$ Merachew W et al. This is an open access article distributed under the Creative Commons Attribution License, which permits unrestricted use, distribution, and reproduction in any medium, provided the original work is properly cited.

Resistance to Triclabendazole was first described in the United Kingdom (UK) in the late 1990's and has now been reported on numerous occasions in fluke populations affecting sheep, and cattle. Triclabendazole resistance is of interest, not only as part of the wider trend of anthelmintic resistance, but also because its appearance presents particular challenges to the management of ruminant livestock, especially sheep, in many areas of the country. Resistance has likely appeared due to a generally poor understanding of liver fluke biology by farmers and con-founding factors, such as incorrect dosing, inappropriate product choice, and lack of testing for efficacy [8].

Mechanisms involved in the development of resistance to the TCBZ can result from changes in the target molecule, in drug uptake/ efflux mechanisms and in drug metabolism [9]. Different methods, both in vivo and in vitro methods, have been used to detect and monitor Triclabendazole resistance. Faecal egg count reduction test is the most used in vivo method and different in vitro methods are described, example; the Egg Hatch Assay (EHA) [10].

A number of strategies have been proposed that may help to avoid or at least slow down the development and spread of TCBZ-R. They include limiting the number of treatments; strategic dosing at particular times of the year, based on epidemiological data; correct dosage; and the annual rotation of anthelmintic, using drugs from different chemical groups. The latter strategy is designed to prevent the build-up of resistance to a particular class of anthelmintic and to minimize the passage of resistance genes early in the selection process. However, a more effective approach is to use combinations of drugs. It is particularly useful when development of resistance reduces the efficacy of an individual drug, but it retains its efficacy in synergistic combinations [11]. Therefore, the objectives of this work were to review Triclabendazole resistance which is currently applicable for the treatment of fasciolosis and to give highlights on the management strategies to combat Triclabendazole drug resistance.

\section{The Disease: Fasciolosis}

Fasciolosis is among the important parasitic diseases in tropical and subtropical countries which limit productivity of ruminants 


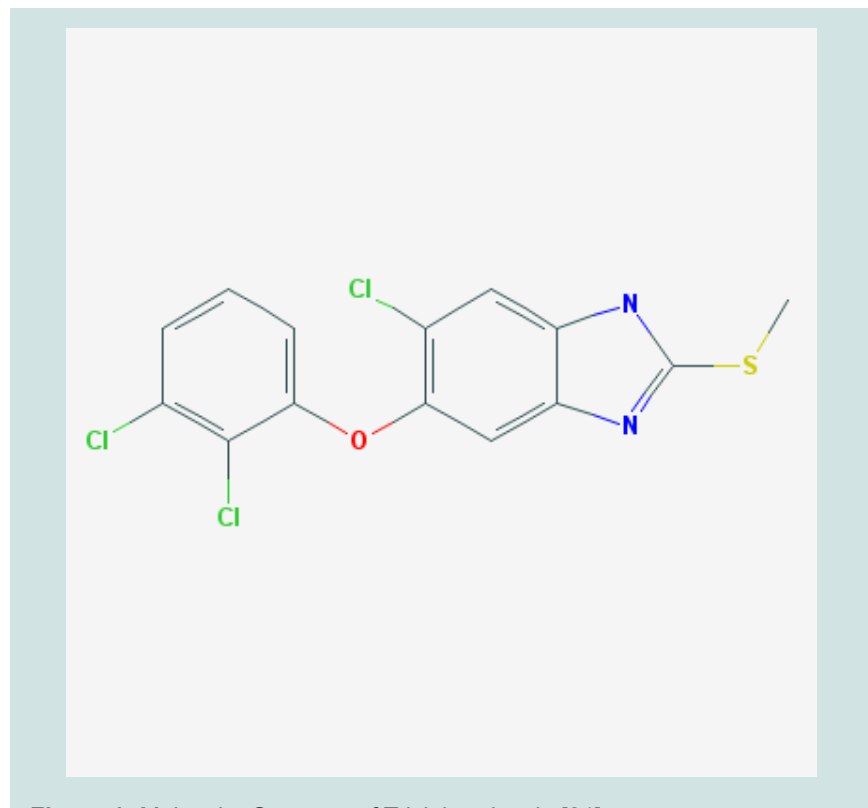

Figure 1: Molecular Structure of Triclabendazole [21].<smiles></smiles>

Figure 2: Chemical Structure of Triclabendazole and its Metabolites [23].

in particular cattle. Fasciola hepatica and F. gigantica are the two liver flukes commonly reported to cause fascioliasis in ruminants [12]. Fasciola spp. infects mammals worldwide, mainly ruminants, but also humans can become infected. In ruminants, and especially in sheep, the infection reduces feed conversion, growth, and meat and milk production. Moreover, it is one of the major causes of liver condemnations at abattoirs and interferes with fertility and fecundity. Fascioliasis is a disease that affects the liver parenchyma and bile ducts of numerous animals, including humans, which causes economic losses and threatens public health [13].

\section{Control and Prevention of Fasciolosis}

Control measures should be done on a preventative rather than curative. Three effective control strategies have been used which are: using of anthelmintic to reduce the number of liver fluke in the definitive hosts and the number of fluke eggs on the pastures, reduce the number of intermediate host and reduce of exposure to infection by managing the fluke prone areas [14].

\section{Use of anthelminthics}

The correct time to use anthelmintics based on weather and climate conditions. Drugs play a crucial role in the control of fascioliasis. More frequent treatments are necessary if you use drugs that are only effective against advanced mature flukes aged 12-16 weeks or older. Using Triclabendazole-based flukicides is the most effective drug against both early mature and adult liver flukes. The best control measures may be achieved if this drug use three times yearly. August/September: to prevent pasture from contamination and to eliminate adult flukes came from autumn and winter. January / February: to completely remove of flukes picked up during late spring and early summer. April/ May: to remove flukes picked up during summer and early autumn [15].

\section{Snail control}

The second available strategy for control of Fasciola spp. is the control of snail as it acts as an intermediate host for the parasite. This can be done by; Chemical control: although chemical control is effective, snails cannot be eradicated by chemicals because they reproduce so readily. Improved drainage: Irrigation projects can provide habitats to the snails. Cleaning of vegetation regularly may



Figure 3: Description; Europe, South America, and Australia left to right, respectively. Global Distribution of Reports of Triclabendazole Resistance (TCBZ-R) in Livestock [34].

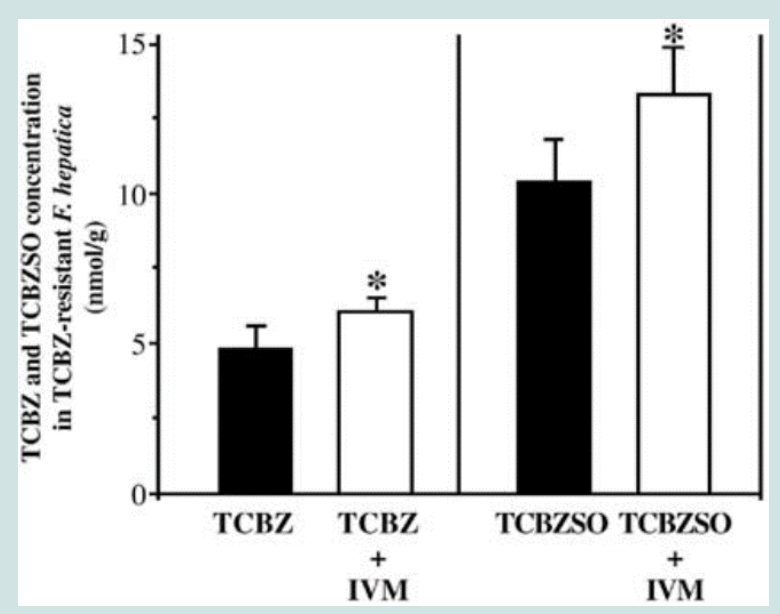

Figure 4: Decreased efflux of TCBZ and TCBZ.SO in TCBZ-resistant flukes following co-incubation with Ivermectin. Where; ${ }^{*}=\mathrm{P}<0.05$ [2]. 


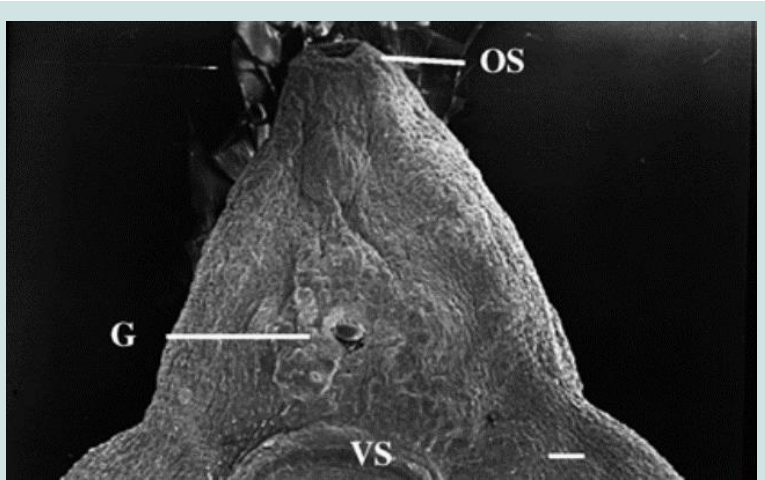

Figure 5: TCBZ-R fluke treated with TCBZSO $(15 \mu \mathrm{g} / \mathrm{ml})$ and verapamil $(1 \times 10-4 \mathrm{M})$ for $24 \mathrm{~h}$. The surface tegument has been removed exposing the underlying basal lamina. OS: Oral sucker, G: gonopore, VS: ventral sucker [2].

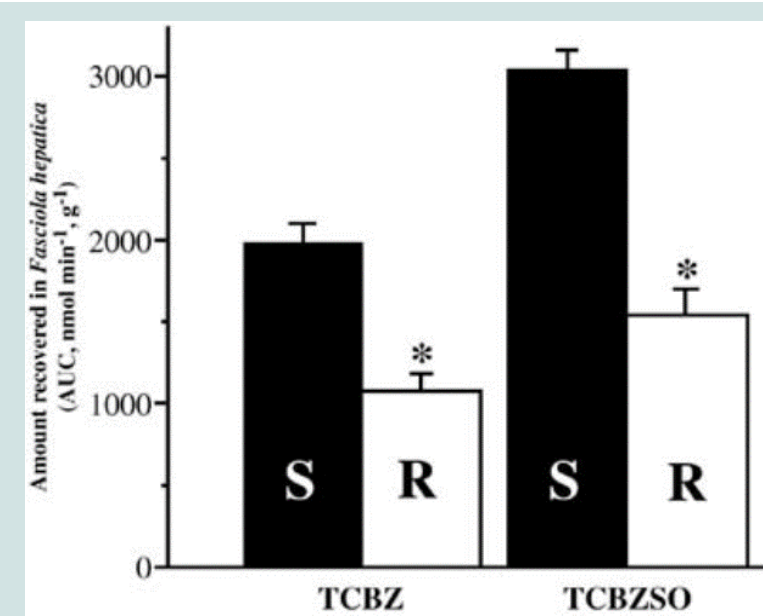

Figure 6: Uptake of TCBZ and TCBZ.SO by TCBZ-S and TCBZ-R flukes Where; ${ }^{*}=\mathrm{P}<0.05[43]$

reduce the contamination of herbage [16].

\section{Disease control by farm management}

This is the third effective strategy for control of Fasciola. This can be accomplished by: Fencing the snail-infested grazing areas consist only a small part of the animals' pasture. Therefore, Fencing off these contaminated areas would be the most economic and efficient method of controlling fascioliasis. Spending a little money on fencing may prevent a serious outbreak of liver fluke disease [17].

\section{The Drug: Triclabendazole}

Effective strategies for the control of fasciolosis are mainly based on the use of drugs. Triclabendazole (Fasinex, Novartis) is worldwide one of the most used drugs for the control of fasciolosis. TCBZ is usually the anthelmintic of choice against $F$. hepatica in livestock, as this drug has high activity against both adult and down to 1 week old juvenile flukes [17]. In animals, TCBZ is the most effective and widely used anthelmintic against immature and mature flukes [8]. Many studies have been conducted on using of TCBZ showing high efficacy against Fasciola spp. However, it has been revealed that in a later study, the significantly low level of efficacies of TCBZ is the indication of resistance of $F$. hepatica against Triclabendazole in sheep [18].

Triclabendazole is the drug of choice in the treatment of fascioliasis. However, in addition to the changing pattern of disease, reports of resistance to TCBZ have appeared in the literature [19], although they may not all represent genuine cases of resistance. Nevertheless, any reports of resistance are a concern, because TCBZ is the only drug that has shown high efficacy against the migratory and juvenile stages of infection to date. Resistance to the drug could potentially set back any recent gains made in the efforts to combat and manage human and animal fascioliasis [20,21].

Triclabendazole is flukicidal BZs compounds extensively used in veterinary medicine, and has excellent activity against mature and immature stages of the liver fluke, F. hepatica. Triclabendazole is able to penetrate the tegument of $F$. hepatica by diffusion, and the fluke is able to sulfoxidate the drug to their sulfoxide metabolite (TCBZSO) [22].

The results conducted by Mottier et al. indicated that the tegument is an important target for TCBZ and albendazol action, and also indicated that TCBZ is better than albendazole in all aspects of the experiments. It could be concluded that TCBZ remains the drug of choice for treating infection with the liver fluke, F. hepatica, and also has become the main drug used to treat animals and human cases $[23,24]$.

\section{Mechanism of action of triclabendazole}

To understand how resistance to TCBZ may develop, it is necessary to understand the mechanism of drug action. TCBZ is a BZs derivative and, by analogy with what is known about other BZs drugs, it would be anticipated that TCBZ might bind to the $\beta$-tubulin molecule and so disrupt microtubule-based processes. Evidence in support of this idea has come from morphological studies on the tegument, vitellaria and testis, following treatment with the active sulphoxide metabolite. For example, there is inhibition of mitosis in the vitelline and spermatogenic cells; disruption of transport processes in the tegument (the outer layer of a trematode), which leads to progressively severe damage of the tegmental surface, culminating in the total loss of the tegument [17].

Loss of tubulin immune-staining in the tegmental syncytium has also been observed the results suggest that the microtubules have disappeared which, in turn, would prevent the movement of secretory bodies from the cell bodies to the tegmental surface. This process is vital for the maintenance of the integrity of the surface membrane and its disruption would explain the severe morphological changes seen [25].

Despite years of research, the precise mode of action of TCBZ is still unclear. TCBZ is a BZ derivative and all available evidence from gastrointestinal round-worms indicates that $\mathrm{BZ}$ anthelmintics bind to $\alpha$ and $\beta$-tubulins within the cells of the parasite, causing disruption of vital processes, such as feeding and digestion. Several morphological studies of the effects of TCBZ and its active metabolites on F. hepatica, have examined the tegument, vitellaria, and testis of the fluke; all three tissues showed significant signs of Ultra structural disruption, consistent with inhibition of microtubule-based processes [26].

There is also a concurrent loss of tubulin immune-staining in the 
Table 1: List of currently available Triclabendazole products and other drugs used to control $F$. hepatica in cattle and sheep worldwide.

\begin{tabular}{|c|c|c|c|}
\hline Flukicides: Active Compound (s) & Method of Administration Available & Age of $\boldsymbol{F}$. hepatica Killed & Reports of Resistance On-Farm \\
\hline TCBZ and TCBZ-based combinations & Oral, pour-on & From early immature \\
\hline Albendazole & Oral, intraruminal & 30 cases \\
\hline Clorsulon & Injectable, oral & From adult; from late immature for oral & 3 cases \\
\hline Closantel & Pour-on, injectable, oral & From late immature \\
\hline Nitrox nil & Injectable & From adult \\
\hline
\end{tabular}

tegmental syncytium, further implicating an interaction with tubulin as the primary mode of action of TCBZ. That said, this has not helped inform our understanding of TCBZ-R, because TCBZ-resistant flukes do not carry the F200Y/E198A or F167Y mutations in $\beta$-tubulin, implicated in $\mathrm{BZ}$ resistance in nematodes, suggesting that alterations to $\beta$-tubulin are not a key component of TCBZ-R [27].

Recently, TCBZ was reported to inhibit adenylate cyclase activity in yeast and/or inhibit the association of GTP-Ras with adenylate cyclase. Most of the studies on the mechanism of action of TCBZ have been carried out with TCBZ.SO. The precise mechanism remains to be fully elucidated, but there is more evidence for an action against microtubules and microtubule-based processes than for other possibilities, such as against energy metabolism or neuromuscular co-ordination [28].

\section{New approaches to understand modes of action of triclabendazole}

The multiplicity of studies reporting different mechanisms of resistance to TCBZ suggests that the mode of action of TCBZ and/or the effects on fluke metabolism are complicated, but the advent of new technologies could allow the target of TCBZ to be unraveled in the foreseeable future. One approach is affinity purification of the putative protein target, whereby TCBZ is immobilized to a solid support and a protein extract is passed over the column, followed by elution of any bound target proteins. This has resulted in the identification of protein targets against several types of drug. However, these methods seem best suited for situations where a high-affinity ligand binds a relatively abundant target protein [29].

A new approach to understanding the mode of action of small molecules is the application of metabolomics, a whole-organism assay approach that identifies metabolic perturbations in a cell upon exposure to drugs. This technique identifies the metabolomics compounds via mass spectrometry or nuclear magnetic resonance and has been applied to several drug studies in various parasites. Thus, a combination of approaches may be required to fully characterize on-target and off-target effects of TCBZ and to clearly define the mechanism(s) of TCBZ action [30].

\section{Triclabendazole Resistance Distribution}

TCBZ (FasinexTM) is the only commercial agent that kills young pathogenic liver fluke, and is considered an Achilles heel in the overall control of liver fluke. Unfortunately, suspected cases of liver fluke parasites resistant to TCBZ have been reported, and without intervention resistance is likely to establish as outbreaks of liver fluke continue to spread [2]. Resistance to Triclabendazole was first described in the UK in the late 1990's and has now been reported on numerous occasions in fluke populations affecting sheep. Exactly how common TCBZ resistance is in different regions of the world not known, but anecdotally it appears to be highly prevalent in fluke populations in sheep rearing areas [4].

Resistance of Triclabendazole described in different parts of the world mostly in European countries such as Netherland, Britain, Russia, Scotland and main land of Europe. The prevalence of Triclabendazole resistance is high in these parts of the world it may be due to more researches have been done in these countries. In these countries Triclabendazole resistance examined by fecal egg count reduction test, egg hatch assay, coproantigen reduction test that indicates the presence of Triclabendazole resistance in those countries [17].

In Britain, there are fewer reports of resistance to TCBZ in fluke populations in cattle, which may reflect the less intensive use of TCBZ in cattle. However resistance was described in 2010 in Scottish beef calves and is becoming more evident as awareness increases. It is important that farmers are warned of the risk of buying in animals carrying resistant fluke populations and take appropriate advice about quarantining animals particularly if coming from fluke endemic parts of the country [31].

In mainland Europe, most reports of TCBZ-R have come from the lower-lying northwestern countries, such as the Netherlands [8]. There are few, if any, reports of confirmed TCBZ-R from central or southern Europe. This most likely reflects the general prevalence of fluke and the perceived need to treat. There is a growing gradient in the prevalence of $F$. hepatica west-to-east and south-to-north in Europe, with prevailing climatic and/or underlying geological conditions probably pivotal. Fox et al. predicted that fluke incidence will increase and spread west-to-east in the UK over the coming decades, based on modeling the Ollerenshaw Indices and UK Climate Projections. Similar trends are predicted to occur across Europe. The implication of this spread of liver fluke is of serious concern in relation to TCBZ-R, since farmers in traditionally fluke-free regions will need to treat animals that may have been exposed to TCBZresistant flukes [32,33].

\section{Risk Factors for triclabendazole resistance}

Resistance has likely appeared due to a generally poor understanding of liver fluke biology by farmers and con-founding factors, such as incorrect dosing, inappropriate product choice, and lack of testing for efficacy. The high frequency of TCBZ use, effectively TCBZ mono therapy with no anthelmintic rotation, was a major contributing factor towards the development of TCBZ-R [8]. Since TCBZ is not a persistent chemical, resistance was likely due to head selection in contrast to tail selection observed with roundworms $[34,35]$.

The failure of TCBZ to kill liver fluke could be due to several factors ranging from problematic drug delivery, reduced host liver metabolism of TCBZ to active pro-drug, or management practices 
that select for TCBZ resistant parasites. The inability of the FECRT to indicate why the drug has failed means that veterinarians cannot fully advise on the spectrum of potential solutions. Thus, current advice if egg counts fail to fall after TCBZ treatment is to switch to an alternative but less effective drug and recommend that TCBZ dosing is suspended to eliminate threat of 'resistant' parasites causing greater production losses [36].

\section{Human cases of triclabendazole resistance}

In recent years, fascioliasis has emerged as a major zoonotic disease, with an increase in the number of human cases, and it is a serious health problem in a number of countries. TCBZ is also the drug of choice for treating fasciolosis in humans and it is conceivable that TCBZ-resistant fluke populations, selected in livestock, could pose a zoonotic risk to human health, especially in areas such as Peru and Bolivia, where there is a high incidence of human infections [37]. The first incidence of TCBZ treatment failure in humans was reported in a livestock farmer in the Netherlands, with further recent reports of four cases from Chile, one case from Turkey, and seven cases from Peru. Clearly, TCBZ-resistant zoonotic infections are a serious emerging issue [38].

\section{Economic impact of triclabendazole resistance}

The economic significance of Triclabendazole resistance is that increasing morbidity and mortality of the animal in addition the capital loss due to the treatment and control of Triclabendazole resistance of fasciolosis. There is also high economic and zoonotic effect of Triclabendazole resistance Fasciola strain if the strain is transmitted to human. An estimate of the total cost of the outbreak of fasciolosis that was compounded by the presence of Triclabendazoleresistant $F$. hepatica was, therefore, approximately $£ 19,200$. This figure corresponds to $£ 8.73$ per ewe, and does not include additional labor costs that were incurred [39].

\section{Modes of Triclabendzole Resistance}

Investigations into the mechanism of resistance to TCBZ have used the Sligo isolate of $F$. hepatica. This isolate has been shown to be resistant to the action of TCBZ in vivo, at both the adult and juvenile stages. Flukes from this isolate also resist the action of TCBZ.SO in vitro, even at abnormally high concentrations [25].

Mechanisms involved in the development of resistance to the TCBZ can result from changes in the target molecule, in drug uptake/ efflux mechanisms and in drug metabolism [9]. With regard to changes in the target molecule, the target is presumed to be $\beta$-tubulin, but tubulin staining is not abolished by TCBZSO in the resistant isolate. However, in nematodes Benzimidazole resistance has been linked to selection of a $\beta$-tubulin isotype with a phenylalanine to tyrosine substitution at position 167 or at position 200. Some amino acid differences have been noted at other positions but whether these amino acid changes are relevant to the resistant phenotype or are due to normal allelic variation in the genes encoding this isotype remains to be determined and many more sequences from individual TCBZsusceptible (TCBZ-S) and -resistant (TCBZ-R) flukes will need to be obtained [40].

Studies are underway in both adult and juvenile fluke to identify the drug-sensitive isotypes by localizing the sites of expression of the various $\alpha$ - and $\beta$-tubulin isotypes, and thus determining which isotypes are expressed in areas that are severely disrupted following TCBZ treatment. At the molecular level, structural studies have shown that the residues that are variable in benzimidazole-resistant organisms are brought together to form a cluster during the folding of the $\beta$-tubulin protein. These also indicated that the cluster of "sensitive" residues was not on the surface of the molecule, raising the question of "how could the drug access this region? [41].

Molecular modeling studies using $\beta$-tubulin sequences from the liver fluke and the nematode Haemonchus contortus have been used to propose a solution. By analogy to the bacterial tubulin homologue FtsZ the angle between the $\mathrm{N}$-terminal, intermediate and C-terminal domains of $\beta$-tubulin was relaxed by $11^{\circ}$. This increased the surface area of the potential benzimidazole binding cleft sufficiently for Triclabendazole to be "docked" in this region. Mammalian and liver fluke tubulins presented a smaller region for binding, commensurate with the restricted effects of Benz imidazole in these organisms [42]. It was proposed that the resistance-conferring mutations at residues 200 and 167 were effective as they allowed the formation of hydrogen bonds "closing off" the binding pocket. The model also suggests that benzimidazoles act not by causing the de-polymerization of microtubules, but by locking the $\beta$ - tubulin moieties in the "open" conformation and thus interfering with the formation of heterodimers with $\alpha$-tubulins prior to microtubule formation. The entry of TCBZ into the fluke has been shown to occur mainly by diffusion across the tegmental syncytium rather than by oral ingestion [24].

The diffusion of both TCBZ and TCBZ.SO into TCBZ-R (Sligo) flukes is significantly lower than in TCBZ-S (Cullompton) flukes [43]. Interestingly, this is not true for the related BZ, albendazole whose uptake is similar in both TCBZ-S and TCBZ-R fluke. The results suggest that the mechanism is specific to TCBZ and that P-glycoprotein-linked drug efflux pumps could potentially be involved in the resistance mechanism. Overexpression of Pgp has been linked to resistance in nematodes to different classes of anthelmintics. Experiments with Pgp inhibitors have shown that it is possible to "reverse" the condition of the flukes, from resistant to susceptible. For example, co-incubation with Ivermectin decreased the efflux of TCBZ and TCBZ.SO in TCBZ-R flukes such that the drug was present at levels comparable to those in TCBZ-S flukes [44].

In contrast, Ivermectin had no impact on the uptake of albendazole in either TCBZ-S or -R flukes. The consequence of Pgp inhibition in TCBZ-R fluke has been demonstrated in a separate morphological study with another Pgp inhibitor, R (+) -verapamil. Co-incubation of $\mathrm{R}(+)$-verapamil plus TCBZ.SO led to severe disruption of the tegument of TCBZ-R flukes, whereas treatment with TCBZ.SO on its own (even at a high concentration) caused minimal changes to the tegmental surface. The disruption to the resistant fluke was comparable to that observed in susceptible flukes following treatment with TCBZ.SO. While a change in efflux pump activity may simply represent a nonspecific mechanism, nevertheless, it is likely to play a significant role in the development of resistance [17].

The identification and localization of the Pgp-linked efflux pumps have yet to be determined. Studies using a laser micro dissection protocol have provided small quantities of specific fluke tissues for Pgp localization. Tegument, gut and reproductive structures have 
been isolated and probed with a Pgp specific primer. The results obtained to date are inconclusive and many more specimens need to be examined. With regard to a role for altered drug metabolism in TCBZ resistance, the sulphoxidation of TCBZ to TCBZ.SO and TCBZSO to the sulphone metabolite (TCBZ.SO2) are both greater in TCBZ-R than -S flukes [45].

Indeed, TCBZ-R flukes have a $39 \%$ greater capacity to metabolize the parent drug. Use of inhibitors has shown that the flavinmonooxygenase (FMO) enzyme system is the main pathway for the metabolism of TCBZ, and it is more important than the cytochrome P450 enzyme system. Moreover, methimazole (MTZ, an FMO inhibitor) had a significantly greater inhibitory impact on TCBZ sulphoxidation in TCBZ-R than -S flukes (43\% as against 34\%). By comparison, the cytochrome $\mathrm{P} 450$ inhibitor, piperonyl butoxide reduced TCBZSO formation to a lesser extent and the inhibition was equal (at 12\%) in the two isolates [43].

\section{Detection of Triclabendazole Resistance}

Different methods, both in vivo and in vitro methods, have been used to detect and monitor AR. Faecal egg count reduction test is the most used in vivo method and gives an estimation of the efficacy of the drug by comparing the egg counts pre and post treatment. The accuracy of the method depends on a correlation between egg counts and worm burdens which is not always present. Different in vitro methods are described. The EHA was first described by Le Jambre for the detection of BZ-resistance. Modification of the original method is developed by Taylor et al. and the method is mostly used for the detection of possible BZ resistance in sheep and horses [46].

\section{In-Vitro method}

The detection of resistance to Triclabendazole (TCBZ) in sheep infected by F. hepatica was studied using an EHA. Fasciola hepatica eggs were recovered from bile and faeces of infected animals by isolates with different grade of anthelmintic resistance to TCBZ: i) a resistant isolate (RT); ii) a susceptible isolate (ST); iii) naturally infected sheep by a susceptible field strain (FST). The EHA is based on the ovicidal properties of some BZs, and on the capacity of eggs from resistant isolates to embrionate and hatch at higher concentrations than those ones from a susceptible isolate [47]. Although the EHA was originally designed to detect AR in Gastrointestinal Nematodes (GIN), some studies have been carried out with $F$. hepatica eggs from gall bladder and/or faeces using TCBZ, Albendazole (ABZ) and their sulphoxide metabolites [48].

A commercial formulation of TCBZ (Fasinex) diluted in Dimethyl Sulfoxide (DMSO) was used to carry out the EHAs. The concentration of TCBZ in this commercial formulation was $50 \mathrm{mg} /$ $\mathrm{ml}$. Dilutions of $10,40,200,1000$ and $5000 \mu \mathrm{g} / \mathrm{ml}$ were prepared to obtain a final concentration in the wells of $0.05,0.2,1,5$, and $25 \mu \mathrm{g} /$ $\mathrm{ml}$ after adding $10 \mu \mathrm{l}$ of each dilution to a total volume of $2 \mathrm{ml}$. In all EHAs, control wells with $10 \mu \mathrm{l}$ of DMSO were included. Eggs from faeces were obtained by sedimentation, from animals infected by ST and from a pool of faeces of sheep naturally infected by FST. Fasciola hepatica eggs were directly recovered from the gall bladder and washed several times with tap water by sedimentation [47].

\section{In-Vivo method}

The main method used to identify TCBZ-R in the field has been the Faecal Egg Count Reduction Test (FECRT), with the recommended post-treatment sample collection time point at 21 days [49]. Other studies using experimental infections have used 14 days for post-treatment sample collection, which may not allow sufficient time for all eggs from dead parasites to pass out of the gall bladder and be excreted [50].

The FECRT is probably most often used, with drug treatment being regarded as successful if there is a $95 \%$ reduction in fluke egg counts by 14 days post-treatment. However, it is known that eggs can be stored in the gall bladder for several weeks, so they may still be present, even though the flukes have been successfully removed; this can lead to false positive results. Moreover, egg production by flukes ceases within 2 days of successful TCBZ treatment [51].

Other disadvantages of the test include the fact that there is no standard method (i.e. sedimentation, floatation, individual or composite samples) and faecal egg counts are not related to fluke numbers; also, for diagnosis of infection, it only detects patent infections and egg shedding is irregular. Fluke counts may be more accurate but are not always carried out and this data runs into problems of trial design and how the flukes are recovered. The FECRT is often used for field cases, though it suffers from the problems outlined above and is not always linked to fluke count data. Controlled clinical trials should be, but are not necessarily always, carried out [52].

\section{Management Strategies to Delay Development of Triclabendazole Resistance}

\section{Use of other drugs and their combinations}

The only chemical options for the control of TCBZ-resistant fluke are, depending on the host species, treatment with clorsulon, nitroxynil, closantel, albendazole, or oxyclozanide [53]. The use of dual-active flukicides has been recommended to control a F. hepatica isolate that was resistant to Triclabendazole and clorsulon when these drugs were administered individually; this isolate was susceptible to these drugs when given as a dual-active formulation. When such formulations have a synergistic effect (i.e., have greater efficacy than the sum of the actives), this may increase the lifespan of the respective actives. Synergy has been seen with several dual-active flukicides (e.g., TCBZ+ clorsulon or TCBZ+ luxabendazole) against TCBZ-resistant fluke in sheep [54].

\section{Vaccines}

An alternative approach to control TCBZ-R would be the development of a livestock vaccine for $F$. hepatica, which would reduce fluke burdens irrespective of the drug-resistance status of the flukes and would not compromise fluke control during lactation. However, no commercial liver fluke vaccine exists, although several experimental vaccines for livestock are under development. No vaccine has shown reproducibly high enough efficacies (> 60\%) in cattle to warrant commercial production, although the leucine aminopeptidase (LAP) vaccine has shown high efficacy (up to 89\%) in sheep [55].

Thus, until a new anthelmintic is developed that kills all developmental stages, including the early immature fluke, a vaccine is the only alternative treatment that could provide ongoing control 
of fluke infections in livestock in regions where TCBZ-R is endemic [56].

\section{Integrated Parasite Management for Farms}

The management practices on farms generally rely solely upon anthelmintics and appear to have contributed to the development of resistance. Management practices must change to preserve the longevity of existing flukicides, because the likelihood of any new flukicides coming to market in the near future is low [55].

Throughout the year, there are periods in which the risk of fluke infection is higher and these periods fluctuate depending upon location and prevailing climatic conditions, but do provide a set of guidelines to determine when treatment may be required. If farmers combine strategic treatments with FECs and the cELISA during high-risk periods, this approach could be used to determine when to drench, which drench to use, or whether treatment is required at all, based on the known thresholds for economic loss [57].

Well-executed strategic treatments will minimize the need for further treatments throughout the year and, therefore, help to preserve the efficacy of existing flukicides. Regular drug efficacy testing, using FECRT and/or CRT, to preserve the efficacy of existing flukicides or TCBZ is essential to allow producers to avoid using products with reduced efficacy and prevent economic losses resulting from unidentified resistance [55].

Flukicides should always be administered according to the product specifications and best-practice methods, which include: weighing individual animals or the heaviest in the herd to determine dose, calibrating drench equipment before use and during treatments, selecting the most potent formulations of product, and, where possible, regularly rotating effective products. In addition, we must also look at how pastures, drinking water, and irrigation can be better managed to decrease the likelihood of $F$. hepatica infection. Pasture management can allocate low-risk pastures (such as newly sown paddocks, hay, or silage paddocks) to young animals during the highrisk periods, to limit the chances of parasite transmission [58].

\section{Conclusion}

In conclusion, livestock production has a great potential to rural farmers in the world. It can be well exploited if fasciolosis and Triclabendazole drug resistance are controlled very well. Triclabendazole drugs are the most realistic means to control animal fasciolosis. However, the increasing trends of Triclabendazole use and Triclabendazole resistance are a serious problem to cattle production in the world. Since there will no new products become available in the near future, it is of utmost important to maintain the efficacy of Triclabendazole. The widespread incidence of TCBZ-R in livestock will be a major threat to global livestock production and producers need see alternative treatments, such as new flukicides or vaccines to control infections. Based on the above conclusion, the following recommendations are forwarded; strict supervision on the usage of Triclabendazole drugs should be implemented; professionals and livestock owners should be well aware of about Triclabendazole drug and its resistance; more attention should be given to the adoption of integrated parasite management strategies in the farms to control the parasite; since there is no literature available on Triclabendazole in
Ethiopia, more researches ought to be done regarding Triclabendazole resistance and its efficacy in various parts of the country.

\section{References}

1. Greenberg RM (2005) $\mathrm{Ca}^{2+}$ signalling, voltage-gated $\mathrm{Ca}^{2+}$ channels and praziquantel in flatworm neuromusculature. Parasitology 131: S97-S108.

2. Brennan GP, Fairweather I, Trudgett A, Hoey E, McConville M, et al. (2007) Understanding triclabendazole resistance. Exp Mol Pathol 82: 104-109.

3. Thomas O, Coles GC, Duffus K (2000) Triclabendazole resistant Fasciola hepatica in south-west Wales. Vet Rec 146: 200.

4. Hodgkinson J, Cwiklinski K, Beesley NJ, Paterson S, Williams DJ (2013) Identification of putative markers of triclabendazole resistance by a genomewide analysis of genetically recombinant Fasciola hepatica. Parasitology 140 : 1523.

5. Sargison ND, Scott PR (2011) Diagnosis and economic consequences of triclabendazole resistance in Fasciola hepatica in a sheep flock in south-east Scotland. Vet Rec 168: 159.

6. McKellar QA, Jackson F (2004) Veterinary anthelmintics: old and new. Trends Parasitol 20: 456-461.

7. Mitreva M, Zarlenga DS, McCarter JP, Jasmer DP (2007) Parasitic nematodes-from genomes to control. Vet Parasitol 148: 31-42.

8. Moll L, Gaasenbeek CP, Vellema P, Borgsteede FH (2000) Resistance of Fasciola hepatica against triclabendazole in cattle and sheep in The Netherlands. Vet Parasitol 91: 153-158.

9. Ouellette M (2001) Biochemical and molecular mechanisms of drug resistance in parasites. Tropical Medicine \& International Health 6: 874-882.

10. McCoy MA, Fairweather I, Brennan GP, Kenny JM, Ellison S, et al. (2005) The efficacy of nitroxynil and triclabendazole administered synchronously against juvenile triclabendazole-resistant Fasciola hepatica in sheep. Res Vet Sci 78(Suppl A): 33

11. Fairweather I, Boray JC (1999) Fasciolicides: efficacy, actions, resistance and its management. The Vet J 158: 81-112.

12. Keyyu JD, Monrad J, Kyvsgaard NC, Kassuku AA (2005) Epidemiology of Fasciola gigantica and amphistomes in cattle on traditional, small-scale dairy and large-scale dairy farms in the southern highlands of Tanzania. Tropical Animal Health and Production 37: 303-314

13. Rojo-Vázquez FA, Meana A, Valcárcel F, Martínez-Valladares M (2012) Update on trematode infections in sheep. Vet Parasitol 189: 15-38.

14. Boray JC, Murray G (1999) Liver fluke disease in sheep and cattle. NSW Agriculture.

15. Kamaludeen J, Graham-Brown J, Stephens N, Miller J, Howell A, et al. (2019) Lack of efficacy of triclabendazole against Fasciola hepatica is present on sheep farms in three regions of England, and Wales. Vet Rec 184: 1-6.

16. Acici M, Bolukbas CS, Gurler AT, Umur S, Buyuktanir O (2013) Seroprevalence of fasciolosis in equines of the Black Sea Region, Turkey. J Equine Vet Sci 33: 62-66.

17. Fairweather I (2005) Triclabendazole: new skills to unravel an old (ish) enigma. J Helminthol 79: 227-234.

18. Aksoy DY, Kerimoglu U, Oto A, Erguven S, Arslan S, et al. Infection with Fasciola hepatica. Clin Microbiol Infect 11: 859-861.

19. Qian LI, Na CH, Yan ZH, Xue-nian XU (2013) Research Progress on Fascioliasis. Chinese Journal of Parasitology and Parasitic Diseases 229234.

20. Mas-Coma S, Valero MA, Bargues MD (2014) Fascioliasis. Adv Exp Med Biol 66: 77-114.

21. National Library of Medicine (NLM) (2018): Triclabendazole Sulfoxide.

22. Alvarez L, Moreno G, Moreno L, Ceballos L, Shaw L, et al. (2009) Comparative assessment of albendazole and triclabendazole ovicidal activity on Fasciola 
hepatica eggs. Vet Parasitol 164: 211-216.

23. Barrera B, Otero JA, Egido E, Prieto JG, Seelig A, et al. (2012) The anthelmintic triclabendazole and its metabolites inhibit the membrane transporter ABCG2/ BCRP. Antimicrob Agents Chemother 56: 3535-3543.

24. Mottier L, Alvarez L, Fairweather I, Lanusse C (2006) Resistance-induced changes in triclabendazole transport in Fasciola hepatica: ivermectin reversal effect. J Parasitol 92: 1355-1360.

25. Robinson MW, Trudgett A, Hoey EM, Fairweather I (2002) Triclabendazoleresistant Fasciola hepatica: beta-tubulin and response to in vitro treatment with triclabendazole. Parasitology 124: 325-338.

26. Fairweather I (2009) Triclabendazole progress report, 2005-2009: an advancement of learning? J Helminthol 83: 139.

27. von Samson-Himmelstjerna G, Walsh TK, Donnan AA, Carriere S, Jackson F et al. (2009) Molecular detection of benzimidazole resistance in Haemonchus contortus using real-time PCR and pyrosequencing. Parasitology 136: 349 358.

28. Lee YJ, Shi R, Witt SN (2013) The small molecule triclabendazole decreases the intracellular level of cyclic AMP and increases resistance to stress in Saccharomyces cerevisiae. PloS One 8: e64337.

29. Trochine A, Alvarez G, Corre S, Faral-Tello P, Durán R, et al. (2014) Trypanosoma cruzi chemical proteomics using immobilized benznidazole. Exp Parasitol 140: 33-38.

30. Creek DJ, Barrett MP (2014) Determination of antiprotozoal drug mechanisms by metabolomics approaches. Parasitol 141: 83-92.

31. Sargison ND, Wilson DJ, Penny CD, Bartley DJ (2010) Unexpected production loss caused by helminth parasites in weaned beef calves. Vet $\operatorname{Rec} 167:$ 752-754.

32. Fox NJ, White PC, McClean CJ, Marion G, Evans A, et al. (2011) Predicting impacts of climate change on Fasciola hepatica risk. PLoS one 6: e16126.

33. Caminade C, van Dijk J, Baylis M, Williams D (2015) Modelling recent and future climatic suitability for fasciolosis in Europe. Geospat Health 9: 301-308.

34. Kelley JM, Elliott TP, Beddoe T, Anderson G, Skuce P, et al. (2016) Current threat of triclabendazole resistance in Fasciola hepatica. Trends Parasitol 32 458-469

35. Fairweather I, Brennan GP, Hanna REB, Robinson MW, Skuce PJ (2020) Drug resistance in liver flukes. Int J Parasitol Drugs Drug Resist 12: 39-59.

36. Morphew RM, Eccleston N, Wilkinson TJ, McGarry J, Perally S, et al (2012) Proteomics and in silico approaches to extend understanding of the glutathione transferase superfamily of the tropical liver fluke Fasciola gigantica. J Proteome Res 11: 5876-5889.

37. Mooney L, Good B, Hanrahan JP, Mulcahy G, de Waal T (2009) The comparative efficacy of four anthelmintics against a natural acquired Fasciola hepatica infection in hill sheep flock in the west of Ireland. Vet Parasitol 164 201-205.

38. Cabada MM, Lopez M, Cruz M, Delgado JR, Hill V, et al. (2016) Treatment failure after multiple courses of triclabendazole among patients with fascioliasis in Cusco, Peru: a case series. PLoS Negl Trop Dis 10: e0004361.

39. Sargison N (2012) Diagnosis of triclabendazole resistance in Fasciola hepatica. Vet Rec 171: 151-152.

40. Wolstenholme AJ, Fairweather I, Prichard R, von Samson-Himmelstjerna G Sangster NC (2004) Drug resistance in veterinary helminths. Trends Parasito 20: 469-476.

41. Downing KH (2000) Structural basis for the action of drugs that affect microtubule dynamics. Emerging Therapeutic Targets 4: 219-237.

42. Robinson MW, Lawson J, Trudgett A, Hoey EM, Fairweather I (2004) The comparative metabolism of triclabendazole sulphoxide by triclabendazolesusceptible and triclabendazole-resistant Fasciola hepatica. Parasitol Res 92: $205-210$.

43. Alvarez LI, Solana HD, Mottier ML, Virkel GL, Fairweather I, et al. (2005)
Altered drug influx/efflux and enhanced metabolic activity in triclabendazoleresistant liver flukes. Parasitology 131: 501-510.

44. Kerboeuf D, Blackhall W, Kaminsky R, von Samson-Himmelstjerna G (2003) P-glycoprotein in helminths: function and perspectives for anthelmintic treatment and reversal of resistance. Int J Antimicrob Agents 22: 332-346.

45. Robinson MW, McFerran N, Trudgett A, Hoey L, Fairweather I (2004) A possible model of benzimidazole binding to $\beta$-tubulin disclosed by invoking an inter-domain movement. J Mol Graph Model 23: 275-284.

46. Taylor MA, Hunt KR, Goodyear KL (2002) Anthelmintic resistance detection methods. Vet Parasitol 103: 183-194.

47. Robles-Pérez D, Martínez-Pérez JM, Rojo-Vázquez FA, Martínez-Valladares M (2015) Screening anthelmintic resistance to triclabendazole in Fasciola hepatica isolated from sheep by means of an egg hatch assay. BMC Vet Res 11: 226.

48. Robles-Pérez D, Martínez-Pérez JM, Rojo-Vázquez FA, Martínez-Valladares M (2013) The diagnosis of fasciolosis in feces of sheep by means of a PCR and its application in the detection of anthelmintic resistance in sheep flocks naturally infected. Vet Parasitol 197: 277-282.

49. Brockwell YM, Elliott TP, Anderson GR, Stanton R, Spithill TW, et al. (2014) Confirmation of Fasciola hepatica resistant to triclabendazole in naturally infected Australian beef and dairy cattle. Int J Parasitol Drugs Drug Resist 4: $48-54$

50. Flanagan A, Edgar HW, Gordon A, Hanna RE, Brennan GP, et al. (2011) Comparison of two assays, a faecal egg count reduction test (FECRT) and a coproantigen reduction test (CRT), for the diagnosis of resistance to triclabendazole in Fasciola hepatica in sheep. Vet Parasitol 176: 170-176.

51. Toner E, Brennan GP, Hanna RE, Edgar HW, Fairweather I (2011) Disruption of egg formation by Fasciola hepatica following treatment in vivo with triclabendazole in the sheep host. Vet Parasitol 177: 79-89.

52. Rokni MB, Massoud J, O'Neill SM, Parkinson M, Dalton JP (2002) Diagnosis of human fasciolosis in the Gilan province of Northern Iran: application of cathepsin L-ELISA. Diagn Microbiol Infect Dis 44: 175-179.

53. Fairweather I (2011) Reducing the future threat from (liver) fluke: realistic prospect or quixotic fantasy? Vet Parasitol 180: 133-143.

54. Martínez-Valladares M, Cordero-Pérez C, Rojo-Vázquez FA (2014) Efficacy of an anthelmintic combination in sheep infected with Fasciola hepatica resistant to albendazole and clorsulon. Exp Parasitol 136: 59-62.

55. Crilly JP, Anderson F, McCormick I, O'Roarke J, Wilson K, et al. (2015) Triclabendazole-resistant liver fluke: issues and strategies. Livestock 20: 8695.

56. Toet H, Piedrafita DM, Spithill TW (2014) Liver fluke vaccines in ruminants: strategies, progress and future opportunities. Int J Parasitol 44: 915-927.

57. Charlier J, Vercruysse J, Morgan E, Van Dijk J, Williams DJL (2014) Recent advances in the diagnosis, impact on production and prediction of Fasciola hepatica in cattle. Parasitology 141: 326-335

58. Coles GC, Jackson F, Pomroy WE, Prichard RK, von Samson-Himmelstjerna $G$, et al. (2006) The detection of anthelmintic resistance in nematodes of veterinary importance. Vet Parasitol 136: 167-185.

\section{Acknowledgement}

Authors would like to express their deepest thanks to God for his permission to do all daily activities as well. Authors also would like to say thanks to Mr. Sileshi Belew for his excellent guidance and advice, valuable simulative suggestions, necessitated encouragements and overall supervisions that highly aid for the completion of this work. 